\title{
Dynamic Analysis of Single-Layered Graphene Nano-Ribbons (SLGNRs) with Variable Cross-Section Resting on Elastic Foundation
}

https://doi.org/10.1515/cls-2019-0011

Received Dec 13, 2018; accepted Jan 24, 2019

\begin{abstract}
This article deals with free vibration of the variable cross-section (non-uniform) single-layered graphene nano-ribbons (SLGNRs) resting on Winkler elastic foundation using the Differential Quadrature Method (DQM). Here characteristic width of the cross-section is varied exponentially along the length of the nano-ribbon while the thickness of the cross section is kept constant. EulerBernoulli beam theory in conjunction with Eringen nonlocal elasticity theory is considered in this study. The numerical as well as graphical results are reported by using MATLAB codes developed by authors. Convergence of present method is explored and our results are compared with known results available in literature showing excellent agreement. Further, effects various parameters on frequency parameters are studied comprehensively.
\end{abstract}

Keywords: Graphene Nano-Ribbons; Eringen's Nonlocal theory; DQM; Winkler Foundation; Variable Cross-Section

\section{Introduction}

Graphene nanoribbons [1-3] are allotropes of carbon made up of narrow strips of graphene having a width less than $50 \mathrm{~nm}$. Mitsutaka Fujita has the credit to introduce theoretical model of Graphene ribbons to analyze the nanoscale effect in graphene [4-6]. The structure of GNR manifest semi-conducting as well as metallic electronic structures having tunable band gaps. GNRs possess all most all the pecuiliar properties Graphene and carbon nanotube along with an additional property viz. tunable band gap. It has low noise, and high thermal and elec-

\footnotetext{
*Corresponding Author: S. Chakraverty: Department of Mathematics, National Institute of Technology, Rourkela-769008, India; Email: sne_chak@yahoo.com

Subrat Kumar Jena: Department of Mathematics, National Institute of Technology, Rourkela-769008, India; Email: sjena430@gmail.com
}

trical conductivities which makes it an alternative of copper regarding integrated circuit systems. Potential application of GNRs include Field effect transistors, LED, Electroactive actuators etc. In this regards, accurate prediction of vibration characteristics of SLGNRs are very essential. For this purpose, various nonclassical continuum theories have been developed but the nonlocal elasticity theory of Eringen [7] is very popular.

Non uniform nanomaterials are very vital in reliable design of various nano devices. One can see several applications of nanomaterials viz. CNT reinforced structure in [8-11]. Also, dynamical behavior of nanobeam, nano-ribbon, nanotube etc. can be found in [12-16]. In the last decades, many studies reported the behavior of non-uniform beams in different static and dynamic manners [17-20]. Peddieson et al. [21] applied Eringen's nonlocal elasticity theory to study Euler-Bernoulli beam. The main output from the model is that MEMS scale devices would not exhibit nonlocal effects whereas nanoscale devices could. Wang et al. [22] applied analytical method to investigate vibration of Euler-Bernoulli and Timoshenko nanobeams. Here rotary inertia and transverse shear deformation were taken into account for Timoshenko nanobeam. One can see bending, buckling and vibration of beam incorporated with nonlocal theories in [23, 24], and Eltaher et al. [25] applied finite element method to study vibration of Euler-Bernoulli nanobeams. Hashemi and Khaniki [26] analytically studied free vibration of a nonlocal nanobeam with variable cross-section.

During the past years, various elastic foundation models such as Winkler, Pasternak, and Flonenko -Borodich foundations have been introduced. It is worth mentioning that the Winkler foundation model was suggested in 1867 by Winkler which has become one of the first scenarios in this regard. Linear algebraic relationship between the normal displacement and the contact pressure [27] is assumed in the modeling of this foundation. This assumption makes it simple to get the closed-form solutions [28, 29]. On the other hand, it also gets a nonlinear behavior with fewer computations in comparison with other meth-

๑ Open Access. ( 2019 S. Kumar Jena and S. Chakraverty, published by De Gruyter mons Attribution 4.0 License 
ods [30-34]. One may see different studies on Winkler elastic foundation in important papers viz. [35, 36]. Finite and infinite beams on a Winkler support have been addressed by Auersch [37]. As regards, Eisenberger and Clastornik [38] studied the vibration and buckling of a beam resting on variable Winkler elastic foundation. Also, the dynamic behavior of infinite beam has been investigated by Ruge and Birk [39]. Next, Lee [40] studied the dynamic response of a Timoshenko beam subject to a moving concentrated mass. Huang and Thambiratnam [41] has used finite strip method to investigate deflection of plates having moving accelerated loads resting on Winkler foundation. Further, $\mathrm{Oz}$ and Pakdemirli [42] have also examined resonances of shallow beams with elastic foundations. Elastic foundations with Winkler-Pasternak problems may also be found in [43, 44]. It may be noted that structural problems with elastic foundation have also been solved by few other methods such as differential transform [45], Rayleigh-Ritz [46] and Harmonic DQ [47, 48].

Though some of the studies have been reported on Winkler elastic foundation, the current article for the first time predicate vibration characteristics of the single-layered graphene nano-ribbons with variable crosssection placed on Winkler elastic foundation.

\section{Mathematical Formulation}

In this study, Eringen [7] nonlocal theory is associated with Euler-Bernoulli beam theory. Here we have considered $\mathrm{x}$ coordinate along the length of the beam, $y$ coordinate along the width of the beam and $\mathrm{z}$ coordinate from the midplane of the beam. One may express strain energy $U$ as

$$
U=\frac{1}{2} \int_{0}^{L} \int_{A} \sigma_{x x} \varepsilon_{x x} d A d x,
$$

where $\sigma_{x x}$ is the normal stress, $L$ is the length and $A$ is the cross-section area. The strain-displacement relation may be given as

$$
\varepsilon_{x x}=-z \frac{\partial^{2} w}{\partial x^{2}}
$$

where $\varepsilon_{x x}$ is the normal strain and $w$ is the deflection function. Substituting Eq. (2) in Eq. (1), one may obtain

$$
U=-\frac{1}{2} \int_{0}^{L} M \frac{\partial^{2} w}{\partial x^{2}} d x,
$$

where $M=\int_{A} z \sigma_{x x} d A$, is the bending moment. In this study, the free harmonic motion is considered, i.e. $w=w_{0}(x) e^{i \omega t}$, where $\omega$ is the natural frequency of vibration. Using free harmonic motion in Eq. (3), we may obtain the strain energy $U$ as

$$
U=-\frac{1}{2} \int_{0}^{L} M \frac{d^{2} w_{0}}{d x^{2}} d x .
$$

The kinetic energy $T$ is given as

$$
T=\frac{1}{2} \int_{0}^{L} \rho A \omega^{2} w_{0}^{2} d x,
$$

where $\rho$ is the mass density and $A$ is the area. Now the potential energy may be given as

$$
V=\frac{1}{2} \int_{0}^{L} f_{e} w_{0} d x,
$$

where $f_{e}$ is the density of reaction force of elastic foundation and is defined as $f_{e}=k_{w} w_{0}$. Here $k_{w}$ denotes the Winkler modulus of the elastic medium. Now by using Hamilton's principle $\int_{0}^{t} \delta T-(\delta U+\delta V) d t=0$ and setting the co-efficient of $\delta w_{0}$ to zero, one may obtain the governing equation as

$$
\frac{d^{2} M}{d x^{2}}-k_{w} w_{0}=-\rho A \omega^{2} w_{0} .
$$

Based on Eringen's nonlocal elasticity theory [7], nonlocal constitutive relation for EBT may be expressed as

$$
M-\mu \frac{d^{2} M}{d x^{2}}=-E I \frac{d^{2} w_{0}}{d x^{2}}
$$

where $\mu$ is the nonlocal parameter which can be expressed as $\mu=\left(e_{0} a\right)^{2}$ with $e_{0}$ and $a$ denoting material constant and internal characteristic length respectively, $I$ is the second moment of area and $E$ is Young's modulus. By using Eq. (7) and Eq. (8), $M$ may be expressed as

$$
M=-E I \frac{d^{2} w_{0}}{d x^{2}}+\mu\left(-\rho A \omega^{2} w_{0}+k_{w} w_{0}\right)
$$

Since we have considered that characteristic width of the cross-section is varied exponentially along the length of the nanoribbon, so from [26]

$$
A(x)=A_{0} e^{n x} \text { and } I(x)=I_{0} e^{n x}
$$

where $n$ is the non-uniform parameter, $I_{0}$ and $A_{0}$ are the second moment of area and cross-section of nanoribbon respectively. Now by using Eq. (9) along with Eq. (10) in Eq. (7), one may obtain the governing equation in terms of displacement as

$$
E I_{0} \frac{d^{4} w_{0}}{d x^{4}}+2 n E I_{0} \frac{d^{3} w_{0}}{d x^{3}}
$$




$$
\begin{aligned}
& +E I_{0}\left(n^{2}+\frac{\rho A_{0} \mu \omega^{2}}{E I_{0}}\right) \frac{d^{2} w_{0}}{d x^{2}}+2 \rho A_{0} \mu \omega^{2} n \frac{d w_{0}}{d x} \\
& +\rho A_{0} \omega^{2} w_{0}\left(\mu n^{2}-1\right)+k_{w} e^{-n x}\left(w_{0}-\mu \frac{d^{2} w_{0}}{d x^{2}}\right)=0
\end{aligned}
$$

We will now introduce non-dimensional terms for easy computation viz. $X=\frac{x}{L}, W=\frac{w_{0}}{L}, \lambda^{2}=\frac{\rho A_{0} \omega^{2} L^{4}}{E I_{0}}, \alpha=$ $\frac{e_{0} a}{L}, K_{w}=\frac{k_{w} L^{4}}{E I_{0}}$ and $\eta=n L$. Substituting the given non-dimensional terms in Eq. (11), we obtain the nondimensionalised form of the governing differential equation as

$$
\begin{aligned}
& \frac{d^{4} W}{d X^{4}}+2 \eta \frac{d^{3} W}{d X^{3}}+\eta^{2} \frac{d^{2} W}{d X^{2}}+K_{w} e^{-\eta x}(W \\
& \left.-\alpha^{2} \frac{d^{2} W}{d X^{2}}\right)=\lambda^{2}\left\{\left(1-\alpha^{2} \eta^{2}\right) W-\alpha^{2} \frac{d^{2} W}{d X^{2}}-2 \alpha^{2} \eta \frac{d W}{d X}\right\}
\end{aligned}
$$

\section{Differential Quadrature Method}

Bellman and Casti [49] have the credit to introduce this technique for the first time. This is an efficient technique to solve both linear and nonlinear equation associated with any physical model. This method is undergone many modifications. In this study we are using Quan and Chang's [50] approach along with Chebyshev-Gauss-Lobatto grid points. For more details of this method one may refer Shu [51].

The derivatives of displacement function $W(X)$ at a given discrete point $i$ can be approximated as weighted sum of functional in the given domain as below.

$$
\begin{aligned}
W_{i}^{\prime} & =\sum_{j=1}^{N} A_{i j} W_{j} \\
W_{i}^{\prime \prime} & =\sum_{j=1}^{N} B_{i j} W_{j} \\
W_{i}^{\prime \prime \prime} & =\sum_{j=1}^{N} C_{i j} W_{j} \\
W_{i}^{I V} & =\sum_{j=1}^{N} D_{i j} W_{j}
\end{aligned}
$$

where $i=1,2, \ldots, N$ and $N$ is the number of discrete grid points.

Here $A_{i j}, B_{i j}, C_{i j}$ and $D_{i j}$ are called the weighting coefficients associated with first, second, third and fourth derivatives respectively.

\subsection{Determination of weighting coefficients}

The weighting coefficient matrix $A=\left(A_{i j}\right)$ can be computed by the following procedure.

For $i \neq j$

$$
\begin{aligned}
& A_{i j}=\frac{1}{X_{j}-X_{i}} \prod_{\substack{k \neq i \\
k \neq j \\
k=1}}^{N} \frac{X_{i}-X_{k}}{X_{j}-X_{k}} \\
& i=1,2, \ldots, N j=1,2, \ldots, N
\end{aligned}
$$

for $i=j$

$$
A_{i i}=\sum_{\substack{k \neq i \\ k=1}}^{N} \frac{1}{X_{i}-X_{k}} \quad i=1,2, \ldots, N .
$$

One may note that the above weighting coefficients are obtained by using Lagrange interpolation polynomial as test function. After getting weighting coefficients of first order derivative, the weighting coefficients of higher order can be obtained as follows.

$$
\begin{gathered}
B=B_{i j}=\sum_{k=1}^{N} A_{i k} A_{k j} \\
C=C_{i j}=\sum_{k=1}^{N} A_{i k} B_{k j} \\
D=D_{i j}=\sum_{k=1}^{N} A_{i k} C_{k j}=\sum_{k=1}^{N} B_{i k} B_{k j} .
\end{gathered}
$$

\subsection{Application of boundary conditions}

Let us now denote

$$
\begin{aligned}
A & =\left[\begin{array}{ccccc}
A_{11} & A_{12} & \cdots & A_{1, N-1} & A_{1, N} \\
A_{21} & A_{22} & \cdots & A_{2, N-1} & A_{2, N} \\
\vdots & \vdots & & \vdots & \vdots \\
A_{N 1} & A_{N 2} & \cdots & A_{N, N-1} & A_{N, N}
\end{array}\right] \\
\bar{A} & =\left[\begin{array}{ccccc}
0 & A_{12} & \cdots & A_{1, N-1} & 0 \\
0 & A_{22} & \cdots & A_{2, N-1} & 0 \\
\vdots & \vdots & & \vdots & \vdots \\
0 & A_{N 2} & \cdots & A_{N, N-1} & 0
\end{array}\right] \\
\bar{A}_{1} & =\left[\begin{array}{cccc}
0 & A_{1,2} & \cdots & A_{1, N} \\
0 & A_{2,2} & \cdots & A_{2, N} \\
\cdots & \cdots & \cdots & \cdots \\
0 & A_{N, 2} & \cdots & A_{N, N}
\end{array}\right] \\
\bar{A}_{2} & =\left[\begin{array}{ccccc}
A_{1,1} & A_{1,2} & \cdots & A_{1, N-1} & 0 \\
A_{2,1} & A_{2,2} & \cdots & A_{2, N-1} & 0 \\
\cdots & \cdots & \cdots & \cdots & \cdots \\
A_{N, 1} & A_{N, 2} & \cdots & A_{N-1, N-1} & 0
\end{array}\right] .
\end{aligned}
$$


Table 1: Validation for S-S case

\begin{tabular}{cccccccccc}
\hline \multirow{2}{*}{$K_{w}$} & \multicolumn{3}{c}{$\sqrt{\lambda_{1}}$} & \multicolumn{3}{c}{$\sqrt{\lambda_{2}}$} & \multicolumn{3}{c}{$\sqrt{\lambda_{3}}$} \\
\cline { 2 - 9 } & Present & {$[45]$} & {$[35]$} & Present & {$[45]$} & {$[35]$} & Present & {$[45]$} & {$[35]$} \\
\hline 10 & 3.2192 & 3.2192 & 3.2192 & 6.2932 & 6.2932 & 6.2932 & 9.4277 & 9.4277 & 9.4277 \\
50 & 3.4844 & 3.4844 & 3.4844 & 6.3329 & 6.3329 & 6.3329 & 9.4396 & 9.4396 & 9.4396 \\
100 & 3.7483 & 3.7483 & 3.7483 & 6.3816 & 6.3816 & 6.3816 & 9.4544 & 9.4544 & 9.4544 \\
200 & 4.1527 & 4.1527 & 4.1527 & 6.4757 & 6.4757 & 6.4757 & 9.4839 & 9.4839 & 9.4839 \\
500 & 4.9438 & 4.9438 & 4.9438 & 6.7358 & 6.7358 & 6.7358 & 9.5706 & 9.5706 & 9.5706 \\
1000 & 5.7556 & 5.7556 & 5.7556 & 7.1121 & 7.1121 & 7.1121 & 9.7101 & 9.7101 & 9.7101 \\
1500 & 6.3219 & 6.3219 & 6.3219 & 7.4366 & 7.4366 & 7.4366 & 9.8439 & 9.8439 & 9.8439 \\
2000 & 6.7673 & 6.7673 & 6.7673 & 7.7235 & 7.7235 & 7.7235 & 9.9724 & 9.9724 & 9.9724 \\
\hline
\end{tabular}

Table 2: Validation for C-C case

\begin{tabular}{|c|c|c|c|c|c|c|}
\hline \multirow[t]{2}{*}{$K_{w}$} & \multicolumn{2}{|c|}{$\sqrt{\lambda_{1}}$} & \multicolumn{2}{|c|}{$\sqrt{\lambda_{2}}$} & \multicolumn{2}{|c|}{$\sqrt{\lambda_{3}}$} \\
\hline & Present & [45] & Present & [45] & Present & [45] \\
\hline 1 & 4.7324 & 4.7324 & 7.8537 & 7.8537 & 10.9958 & 10.9958 \\
\hline 10 & 4.7534 & 4.7534 & 7.8583 & 7.8583 & 10.9975 & 10.9975 \\
\hline 100 & 4.9503 & 4.9503 & 7.9043 & 7.9043 & 11.0144 & 11.0144 \\
\hline 1000 & 6.2239 & 6.2239 & 8.3251 & 8.3251 & 11.1790 & 11.1790 \\
\hline
\end{tabular}

Now, the weighting coefficients of higher order derivatives for different edge conditions are given below.

\section{Simply Supported-Simply Supported}

$$
\begin{aligned}
\left\{W^{\prime}\right\} & =[\bar{A}]\{W\} \\
\left\{W^{\prime \prime}\right\} & =[A]\left\{W^{\prime}\right\}=[A][\bar{A}]\{W\}=[\bar{B}]\{W\} \\
\left\{W^{\prime \prime \prime}\right\} & =[\bar{A}]\left\{W^{\prime \prime}\right\}=[\bar{A}][\bar{B}]\{W\}=[\bar{C}]\{W\} \\
\left\{W^{I V}\right\} & =[A]\left\{W^{\prime \prime \prime}\right\}=[A][\bar{C}]\{W\}=[\bar{D}]\{W\}
\end{aligned}
$$

\section{Clamped-Simply Supported}

$$
\begin{aligned}
\left\{W^{\prime}\right\} & =[\bar{A}]\{W\} \\
\left\{W^{\prime \prime}\right\} & =\left[\bar{A}_{1}\right]\left\{W^{\prime}\right\}=\left[\bar{A}_{1}\right][\bar{A}]\{W\}=[\bar{B}]\{W\} \\
\left\{W^{\prime \prime \prime}\right\} & =\left[\bar{A}_{2}\right]\left\{W^{\prime \prime}\right\}=\left[\bar{A}_{2}\right][\bar{B}]\{W\}=[\bar{C}]\{W\} \\
\left\{W^{I V}\right\} & =[A]\left\{W^{\prime \prime \prime}\right\}=[A][\bar{C}]\{W\}=[\bar{D}]\{W\}
\end{aligned}
$$

\section{Clamped-Clamped}

$$
\begin{aligned}
\left\{W^{\prime}\right\} & =[\bar{A}]\{W\} \\
\left\{W^{\prime \prime}\right\} & =[\bar{A}]\left\{W^{\prime}\right\}=[\bar{A}][\bar{A}]\{W\}=[\bar{B}]\{W\} \\
\left\{W^{\prime \prime \prime}\right\} & =[A]\left\{W^{\prime \prime}\right\}=[A][\bar{B}]\{W\}=[\bar{C}]\{W\} \\
\left\{W^{I V}\right\} & =[A]\left\{W^{\prime \prime \prime}\right\}=[A][\bar{C}]\{W\}=[\bar{D}]\{W\}
\end{aligned}
$$

\section{Clamped-Free}

$$
\begin{aligned}
\left\{W^{\prime}\right\} & =\left[\bar{A}_{1}\right]\{W\} \\
\left\{W^{\prime \prime}\right\} & =\left[\bar{A}_{1}\right]\left\{W^{\prime}\right\}=\left[\bar{A}_{1}\right]\left[\bar{A}_{1}\right]\{W\}=[\bar{B}]\{W\}
\end{aligned}
$$

$$
\begin{aligned}
& \left\{W^{\prime \prime \prime}\right\}=\left[\bar{A}_{2}\right]\left\{W^{\prime \prime}\right\}=\left[\bar{A}_{2}\right][\bar{B}]\{W\}=[\bar{C}]\{W\} \\
& \left\{W^{I V}\right\}=\left[\bar{A}_{2}\right]\left\{W^{\prime \prime \prime}\right\}=\left[\bar{A}_{2}\right][\bar{C}]\{W\}=[\bar{D}]\{W\}
\end{aligned}
$$

Substituting the value of Eq. (13) into Eq. (12), one may obtain generalized eigenvalue problem as

$$
[S]\{W\}=\lambda^{2}[T]\{W\}
$$

where $S$ is the stiffness matrix and $T$ is the mass matrix.

\section{Numerical results and discussions}

MATLAB program is used to solve Eq. (19) and frequency parameters $\sqrt{\lambda}$ have been obtained. Following parameters are taken from for computational purpose.

$E=1 \mathrm{TPa}, L=10 \mathrm{~nm}, h=1$ and unless mentioned $\frac{L}{h}=10$.

\subsection{Validation}

To verify the correctness of present method, we have adopted two ways. Firstly, we consider the SLGNR with nonlocal parameter $\mu=0$ and non-uniform parameter $\eta=$ 0 . We compare our results of frequency parameter $(\sqrt{\lambda})$ with those available in the literature $[35,45]$ for different 
Table 3: Validation for C-F case

\begin{tabular}{|c|c|c|c|c|c|c|}
\hline \multirow[t]{2}{*}{$K_{w}$} & \multicolumn{2}{|c|}{$\sqrt{\lambda_{1}}$} & \multicolumn{2}{|c|}{$\sqrt{\lambda_{2}}$} & \multicolumn{2}{|c|}{$\sqrt{\lambda_{3}}$} \\
\hline & Present & [45] & Present & [45] & Present & [45] \\
\hline 1 & 1.9119 & 1.9119 & 4.6965 & 4.6965 & 7.8552 & 7.8552 \\
\hline 10 & 2.1746 & 2.1746 & 4.7180 & 4.7180 & 7.8599 & 7.8599 \\
\hline 100 & 3.2557 & 3.2557 & 4.9191 & 4.9191 & 7.9058 & 7.9058 \\
\hline 1000 & 5.6407 & 5.6407 & 6.2082 & 6.2082 & 8.3264 & 8.3264 \\
\hline
\end{tabular}

Table 4: Validation for S-S case

\begin{tabular}{ccccc}
\hline$\mu$ & Present & {$[23]$} & {$[24]$} & {$[25]$} \\
\hline 0 & 9.8696 & 9.8696 & 9.8696 & 9.8696 \\
1 & 9.4159 & 9.4159 & 9.4124 & 9.4159 \\
2 & 9.0195 & 9.0195 & 9.0133 & 9.0195 \\
3 & 8.6693 & 8.6693 & 8.6611 & 8.6693 \\
4 & 8.3569 & 8.3569 & 8.3472 & 8.3569 \\
\hline
\end{tabular}

Winkler modulus parameter and are given in Tables 1-3. Secondly, we consider the SLGNR as uniform and is not placed on any elastic foundation viz. $\eta=0$ and $K_{w}=0$. Validation of fundamental frequency parameters $(\lambda)$ which are obtained by present method is accomplished by comparing with the results available in the literature [23-25] which are depicted in Tables 4. Again comparison of presnt results are achieved with [22] for different $\alpha=\frac{e_{0} a}{L}$ which are reported in Tables 5-8. These Tables 1-8 shows the evidence of correctness of present results with those available in the literature.

\subsection{Convergence}

Convergence of the prent study is explored by varying grid points with frequency parameters which are demonstrated in Figure 1 and in Tables 9-12. All the standard boundary conditions such as S-S, C-S, C-C and C-F are taken into consideration with $L=10, \eta=1, \mu=1$ and $K_{w}=10$. From these results, it is very clear that convergence is achieving fast with an increase in a number of grid points and twelve grid points are sufficient to compute the desired results.

\subsection{Effect of nonlocal parameter}

The impacts of nonlocal parameters on frequency parameters are noted in this subsection. The nonlocal parameters are allowed to vary from 0 to $1 \mathrm{~nm}^{2}$ with an increment of 0.2 . All the standard boundary conditions such as S-S, C-S, C-C, and $\mathrm{C}-\mathrm{F}$ are taken into investigation with non-uniform pa- rameter $(\eta)$ as 0.5 , Winkler modulus parameter $K_{w}=50$ and $L=10 \mathrm{~nm}$. All the tabular and graphical results are demonstrated in Figure 2 and Tables 13-16. These results reveal that frequency parameters decrease with increase in nonlocal parameter except first fundamental frequency parameter of $\mathrm{CF}$ nanobeams and $\mathrm{CC}$ nanobeams possess having the highest frequency parameters among all other boundary conditions taken into investigation.

\subsection{Effect of non-uniform parameter}

This subsection deals with the variation of non-uniform parameter $\eta$ on frequency parameters. The non-uniform parameters are allowed to vary from -2 to 2 with an increment of 1. Tables 17-20 and Figure 3 illustrate the response of frequency parameter with non-uniform parameter for all the standard edges and these computations are accomplished with $\mu=1 \mathrm{~nm}^{2}, L=10$ and $K_{w}=500$. One observation regarding the non-uniform parameters is that the frequency parameter decreases with increase in the nonuniform parameter and this decrease is more significant in case of higher modes.

\subsection{Effect of aspect ratio}

The effect of aspect ratio $(\mathrm{L} / \mathrm{h})$ is reported for all the standard boundary conditions for different $(10,20,30,40,50)$. Figure 4 and Tables 21-24 represent the response in term of the graphical and tabular results which are accomplished by taking $\mu=1, K_{w}=50$ and. One important point is to note that frequency parameters with increase in aspect ratio for all the mode of all the standard boundary conditions except $1^{\text {st }}$ mode of C-F case, where it is just reverse.

\subsection{Effect of Winkler modulus parameter}

Effect of Winkler modulus parameter $\left(K_{w}\right)$ on the first four frequency parameters has been studied. First four frequency parameters of all the classical boundary condi- 
Table 5: Validation for S-S case

\begin{tabular}{|c|c|c|c|c|c|c|c|c|c|c|c|}
\hline \multirow{2}{*}{$\alpha=e_{0} a / L$} & \multirow{2}{*}{$\begin{array}{l}\frac{0}{0} \\
\sum\end{array}$} & \multicolumn{2}{|c|}{$1^{\text {st }}$ Mode } & \multicolumn{2}{|c|}{$2^{\text {nd }}$ Mode } & \multicolumn{2}{|c|}{$3^{\text {rd }}$ Mode } & \multicolumn{2}{|c|}{$4^{\text {th }}$ Mode } & \multicolumn{2}{|c|}{$5^{\text {th }}$ Mode } \\
\hline & & Present & {$[22]$} & Present & [22] & Present & [22] & Present & [22] & Present & {$[22]$} \\
\hline 0 & & 3.1416 & 3.1416 & 6.2832 & 6.2832 & 9.4248 & 9.4248 & 1.566 & 1.566 & 15.708 & 15.708 \\
\hline 0.1 & & 3.0685 & 3.0685 & 5.7817 & 5.7817 & 8.0400 & 8.0400 & 9.9161 & 9.9161 & 11.5111 & 11.5111 \\
\hline 0.3 & & 2.6800 & 2.6800 & 4.3013 & 4.3013 & 5.4422 & 5.4422 & 6.3630 & 6.3630 & 7.1568 & 7.1568 \\
\hline
\end{tabular}

Table 6: Validation for C-S case

\begin{tabular}{|c|c|c|c|c|c|c|c|c|c|c|}
\hline \multirow{2}{*}{$\alpha=e_{0} a / L$} & \multicolumn{2}{|c|}{$1^{\text {st }}$ Mode } & \multicolumn{2}{|c|}{$2^{\text {nd }}$ Mode } & \multicolumn{2}{|c|}{$3^{\text {rd }}$ Mode } & \multicolumn{2}{|c|}{$4^{\text {th }}$ Mode } & \multicolumn{2}{|c|}{$5^{\text {th }}$ Mode } \\
\hline & Present & {$[22]$} & Present & {$[22]$} & Present & [22] & Present & [22] & Present & [22] \\
\hline 0 & 3.9266 & 3.9266 & 7.0686 & 7.0686 & 10.2102 & 10.2102 & 13.3518 & 13.3518 & 16.4934 & 16.4934 \\
\hline 0.1 & 3.8209 & 3.8209 & 6.4649 & 6.4649 & 8.6517 & 8.6 & 10.469 & 10.4 & 12.018 & 12.018 \\
\hline 0.3 & 3.2828 & 3.2828 & 4.7668 & 4.7668 & 5.8371 & 5.8371 & 6.7143 & 6.7143 & 7.4773 & 7.4773 \\
\hline
\end{tabular}

Table 7: Validation for C-C case

\begin{tabular}{|c|c|c|c|c|c|c|c|c|c|c|}
\hline \multirow{2}{*}{$\alpha=e_{0} a / L$} & \multicolumn{2}{|c|}{$1^{\text {st }}$ Mode } & \multicolumn{2}{|c|}{$2^{\text {nd }}$ Mode } & \multicolumn{2}{|c|}{$3^{\text {rd }}$ Mode } & \multicolumn{2}{|c|}{$4^{\text {th }}$ Mode } & \multicolumn{2}{|c|}{$5^{\text {th }}$ Mode } \\
\hline & Present & [22] & Present & [22] & Present & [22] & Present & [22] & Present & [22] \\
\hline 0 & 4.7300 & 4.7300 & 7.8532 & 7.8532 & 10.9956 & 10.9956 & 14.1372 & 14.1372 & 17.2787 & 17.2787 \\
\hline 0.1 & 4.5945 & 4.5945 & 7.1402 & 7.1402 & 9.2583 & 9.2583 & 11.016 & 11.016 & 12.520 & 12.520 \\
\hline 0.3 & 3.9184 & 3.9184 & 5.1963 & 5.1963 & 6.2317 & 6.2317 & 7.0482 & 7.0482 & 7.7955 & 7.7955 \\
\hline
\end{tabular}

Table 8: Validation for C-F case

\begin{tabular}{|c|c|c|c|c|c|c|c|c|c|c|c|}
\hline \multirow{2}{*}{\multicolumn{2}{|c|}{$\alpha=e_{0} a / L \quad \stackrel{0}{\stackrel{0}{\circ}}$}} & \multicolumn{2}{|c|}{$1^{\text {st }}$ Mode } & \multicolumn{2}{|c|}{$2^{\text {nd }}$ Mode } & \multicolumn{2}{|c|}{$3^{\text {rd }}$ Mode } & \multicolumn{2}{|c|}{$4^{\text {th }}$ Mode } & \multicolumn{2}{|c|}{$5^{\text {th }}$ Mode } \\
\hline & & Present & [22] & Present & [22] & Present & [22] & Present & [22] & Present & [22] \\
\hline 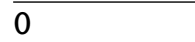 & & 1.8751 & 1.8751 & 4.6941 & 4.6941 & 7.8548 & 7.8548 & 10.9955 & 10.9955 & 14.1372 & 14.1372 \\
\hline 0.1 & & 1.8792 & 1.8792 & 4.5475 & 4.54 & 7.1459 & 7.1 & 9.2569 & 9.2 & 11. & 11.016 \\
\hline 0.3 & & 1.9154 & 1.9154 & 3.7665 & 3.7665 & 5.2988 & 5.2988 & 6.1385 & 6.1385 & 7.1450 & 7.1450 \\
\hline
\end{tabular}

Table 9: Response of frequency parameters with grid points for SS case

\begin{tabular}{ccccc}
\hline$N$ & $\sqrt{\lambda_{1}}$ & $\sqrt{\lambda_{2}}$ & $\sqrt{\lambda_{3}}$ & $\sqrt{\lambda_{4}}$ \\
\hline 3 & 2.7361 & - & - & - \\
4 & 3.1797 & 5.2502 & - & - \\
5 & 3.0783 & 6.2776 & 7.6390 & - \\
6 & 3.0861 & 5.7216 & 9.1149 & 9.9562 \\
7 & 3.0863 & 5.7979 & 7.8481 & 11.6940 \\
8 & 3.0863 & 5.7956 & 8.0757 & 9.5864 \\
10 & 3.0863 & 5.7956 & 8.0510 & 9.9058 \\
12 & 3.0863 & 5.7957 & 8.0502 & 9.9229 \\
14 & 3.0863 & 5.7957 & 8.0502 & 9.9235 \\
16 & 3.0863 & 5.7957 & 8.0502 & 9.9235 \\
\hline
\end{tabular}

Table 10: Response of frequency parameters with grid points for CS case

\begin{tabular}{ccccc}
\hline$N$ & $\sqrt{\lambda_{1}}$ & $\sqrt{\lambda_{2}}$ & $\sqrt{\lambda_{3}}$ & $\sqrt{\lambda_{4}}$ \\
\hline 3 & 2.4936 & - & - & - \\
4 & 3.4239 & 4.7799 & - & - \\
5 & 3.6867 & 6.2257 & 7.1386 & - \\
6 & 3.7116 & 6.2895 & 9.1574 & 9.5255 \\
8 & 3.7081 & 6.4102 & 8.6762 & 10.0479 \\
10 & 3.7082 & 6.4159 & 8.6182 & 10.3505 \\
12 & 3.7082 & 6.4159 & 8.6213 & 10.4493 \\
14 & 3.7082 & 6.4159 & 8.6210 & 10.4469 \\
16 & 3.7082 & 6.4159 & 8.6210 & 10.4472 \\
18 & 3.7082 & 6.4159 & 8.6210 & 10.4472 \\
\hline
\end{tabular}




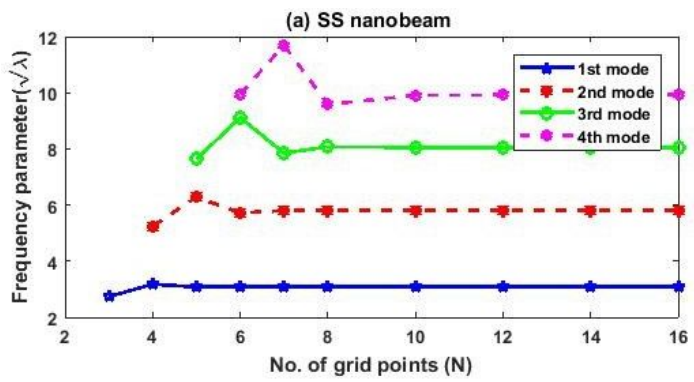

(c) CC nanobeam

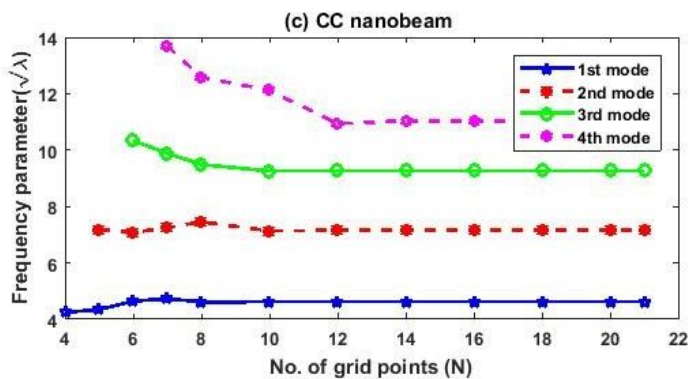

Figure 1: Frequency parameters Vs. grid points

Table 11: Response of frequency parameters with grid points for CC case

\begin{tabular}{ccccc}
\hline$N$ & $\sqrt{\lambda_{1}}$ & $\sqrt{\lambda_{2}}$ & $\sqrt{\lambda_{3}}$ & $\sqrt{\lambda_{4}}$ \\
\hline 4 & 4.2431 & - & - & - \\
5 & 4.3489 & 7.1782 & - & - \\
6 & 4.6461 & 7.0825 & 10.3331 & - \\
7 & 4.7300 & 7.2439 & 9.8880 & 13.6930 \\
8 & 4.6180 & 7.4575 & 9.4901 & 12.5849 \\
10 & 4.6208 & 7.1344 & 9.2584 & 12.1193 \\
12 & 4.6208 & 7.1576 & 9.2702 & 10.9375 \\
14 & 4.6208 & 7.1561 & 9.2689 & 11.0426 \\
16 & 4.6208 & 7.1562 & 9.2690 & 11.0210 \\
18 & 4.6208 & 7.1562 & 9.2690 & 11.0231 \\
20 & 4.6208 & 7.1562 & 9.2690 & 11.0230 \\
21 & 4.6208 & 7.1562 & 9.2690 & 11.0230 \\
\hline
\end{tabular}

Table 12: Response of frequency parameters with grid points for CF case

\begin{tabular}{ccccc}
\hline$N$ & $\sqrt{\lambda_{1}}$ & $\sqrt{\lambda_{2}}$ & $\sqrt{\lambda_{3}}$ & $\sqrt{\lambda_{4}}$ \\
\hline 3 & 1.8796 & 2.5457 & - & - \\
4 & 2.0202 & 3.8591 & 4.5134 & - \\
6 & 2.0466 & 4.5329 & 6.7832 & 9.7516 \\
8 & 2.0465 & 4.5053 & 7.1253 & 9.3260 \\
10 & 2.0465 & 4.5064 & 7.1407 & 9.2508 \\
12 & 2.0465 & 4.5063 & 7.1407 & 9.2596 \\
14 & 2.0465 & 4.5063 & 7.1407 & 9.2589 \\
16 & 2.0465 & 4.5063 & 7.1407 & 9.2589 \\
\hline
\end{tabular}
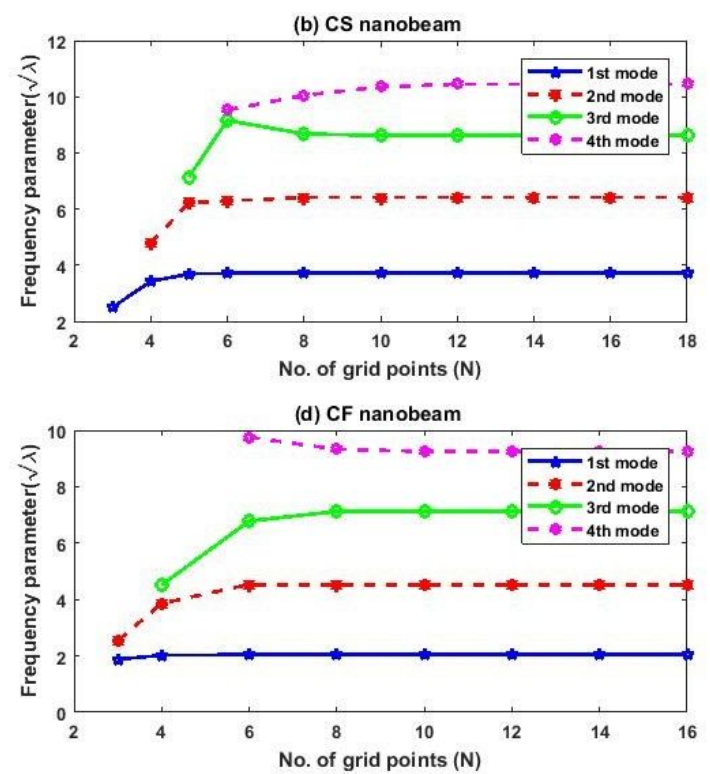

Table 13: Response of frequency parameter on nonlocal parameter for SS case

\begin{tabular}{ccccc}
\hline$\mu$ & $\sqrt{\lambda_{1}}$ & $\sqrt{\lambda_{2}}$ & $\sqrt{\lambda_{3}}$ & $\sqrt{\lambda_{4}}$ \\
\hline 0 & 3.3586 & 6.3153 & 9.4357 & 12.5718 \\
1 & 3.2999 & 5.8227 & 8.0566 & 9.9254 \\
2 & 3.2483 & 5.4814 & 7.3224 & 8.8122 \\
3 & 3.2025 & 5.2246 & 6.8371 & 8.1345 \\
4 & 3.1616 & 5.0213 & 6.4813 & 7.6583 \\
5 & 3.1248 & 4.8544 & 6.2039 & 7.2965 \\
\hline
\end{tabular}

Table 14: Response of frequency parameter on nonlocal parameter for CS case

\begin{tabular}{ccccc}
\hline$\mu$ & $\sqrt{\lambda_{1}}$ & $\sqrt{\lambda_{2}}$ & $\sqrt{\lambda_{3}}$ & $\sqrt{\lambda_{4}}$ \\
\hline 0 & 3.9850 & 7.0573 & 10.1949 & 13.3380 \\
1 & 3.8908 & 6.4640 & 8.6451 & 10.4625 \\
2 & 3.8088 & 6.0622 & 7.8397 & 9.2792 \\
3 & 3.7364 & 5.7640 & 7.3128 & 8.5634 \\
4 & 3.6721 & 5.5299 & 6.9285 & 8.0618 \\
5 & 3.6143 & 5.3389 & 6.6298 & 7.6812 \\
\hline
\end{tabular}

tions such as SS, CS, CC and CF are given in Tables 25-28 for different $K_{w}$. In this table, $\mu=1 \mathrm{~nm}^{2}, L=20$ and $\eta=0.5$ are taken into consideration. Graphical results are depicted in Figure 6 where variation of first four frequency parameters with has been shown. In this figure, the value of $\left(K_{w}\right)$ is given as $0,50,100,200,500,1000$. 


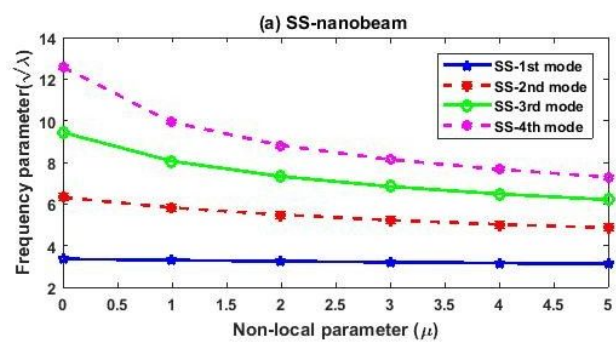

(c) CC-nanobeam

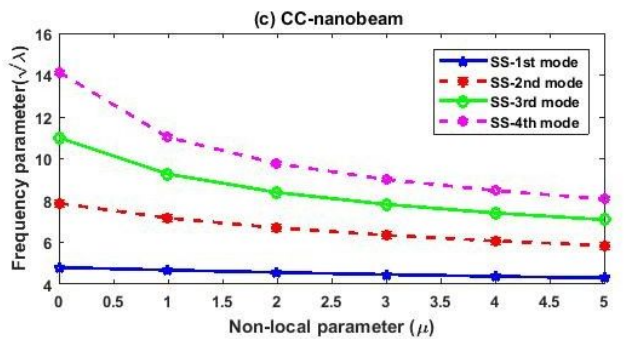

Figure 2: Frequency parameters Vs nonlocal parameters

Table 15: Response of frequency parameter on nonlocal parameter for CC case

\begin{tabular}{ccccc}
\hline$\mu$ & $\sqrt{\lambda_{1}}$ & $\sqrt{\lambda_{2}}$ & $\sqrt{\lambda_{3}}$ & $\sqrt{\lambda_{4}}$ \\
\hline 0 & 4.8035 & 7.8717 & 11.0036 & 14.1417 \\
1 & 4.6747 & 7.1643 & 9.2702 & 11.0230 \\
2 & 4.5625 & 6.6915 & 8.3888 & 9.7636 \\
3 & 4.4637 & 6.3434 & 7.8181 & 9.0056 \\
4 & 4.3757 & 6.0715 & 7.4044 & 8.4753 \\
5 & 4.2966 & 5.8505 & 7.0841 & 8.0731 \\
\hline
\end{tabular}

Table 16: Response of frequency parameter on nonlocal parameter for $\mathrm{CF}$ case

\begin{tabular}{ccccc}
\hline$\mu$ & $\sqrt{\lambda_{1}}$ & $\sqrt{\lambda_{2}}$ & $\sqrt{\lambda_{3}}$ & $\sqrt{\lambda_{4}}$ \\
\hline 0 & 2.5691 & 4.7267 & 7.8407 & 10.9779 \\
1 & 2.5825 & 4.6094 & 7.1657 & 9.2693 \\
2 & 2.5965 & 4.4986 & 6.7124 & 8.3850 \\
3 & 2.6110 & 4.3929 & 6.3838 & 7.8005 \\
4 & 2.6262 & 4.2916 & 6.1351 & 7.3644 \\
5 & 2.6422 & 4.1940 & 5.9423 & 7.0144 \\
\hline
\end{tabular}

Table 17: Response of frequency parameter with non-uniform parameter for SS case

\begin{tabular}{ccccc}
\hline$\eta$ & $\sqrt{\lambda_{1}}$ & $\sqrt{\lambda_{2}}$ & $\sqrt{\lambda_{3}}$ & $\sqrt{\lambda_{4}}$ \\
\hline-2 & 7.8944 & 8.3196 & 9.4262 & 10.7616 \\
-1 & 6.1705 & 7.0580 & 8.6320 & 10.2525 \\
0 & 4.9256 & 6.3416 & 8.2704 & 10.0418 \\
1 & 4.0576 & 6.0143 & 8.1351 & 9.9691 \\
2 & 3.5003 & 5.9032 & 8.1059 & 9.9589 \\
\hline
\end{tabular}

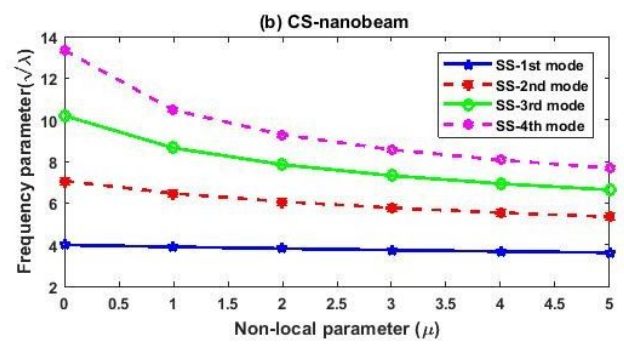

(d) CF-nanobeam

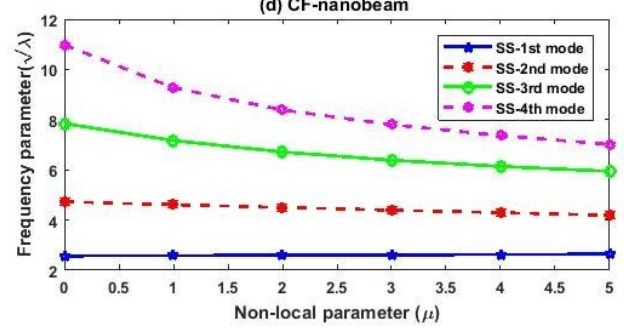

Table 18: Response of frequency parameter with non-uniform parameter for CS case

\begin{tabular}{ccccc}
\hline$\eta$ & $\sqrt{\lambda_{1}}$ & $\sqrt{\lambda_{2}}$ & $\sqrt{\lambda_{3}}$ & $\sqrt{\lambda_{4}}$ \\
\hline-2 & 7.9868 & 8.6471 & 9.8887 & 11.2541 \\
-1 & 6.3321 & 7.5132 & 9.1760 & 10.7849 \\
0 & 5.1676 & 6.8847 & 8.8385 & 10.5760 \\
1 & 4.3842 & 6.5799 & 8.6904 & 10.4864 \\
2 & 3.8861 & 6.4472 & 8.6322 & 10.4519 \\
\hline
\end{tabular}

Table 19: Response of frequency parameter with non-uniform parameter for CC case

\begin{tabular}{ccccc}
\hline$\eta$ & $\sqrt{\lambda_{1}}$ & $\sqrt{\lambda_{2}}$ & $\sqrt{\lambda_{3}}$ & $\sqrt{\lambda_{4}}$ \\
\hline-2 & 8.0735 & 8.9193 & 10.2689 & 11.6639 \\
-1 & 6.5266 & 7.9405 & 9.6671 & 11.2670 \\
0 & 5.5453 & 7.4613 & 9.4119 & 11.1081 \\
1 & 5.0224 & 7.2759 & 9.3250 & 11.0564 \\
2 & 4.8224 & 7.2390 & 9.3174 & 11.0543 \\
\hline
\end{tabular}

Table 20: Response of frequency parameter with non-uniform parameter for CF case

\begin{tabular}{ccccc}
\hline$\eta$ & $\sqrt{\lambda_{1}}$ & $\sqrt{\lambda_{2}}$ & $\sqrt{\lambda_{3}}$ & $\sqrt{\lambda_{4}}$ \\
\hline-2 & 7.8762 & 7.9678 & 8.8392 & 10.1762 \\
-1 & 6.0870 & 6.4752 & 7.9127 & 9.6349 \\
0 & 4.7579 & 5.5188 & 7.4662 & 9.4105 \\
1 & 3.7891 & 4.9497 & 7.2652 & 9.3162 \\
2 & 2.9990 & 4.5773 & 7.1568 & 9.2583 \\
\hline
\end{tabular}


Table 21: Response of frequency parameter with length-to-height ratio for SS case

\begin{tabular}{ccccc}
\hline$L / h$ & $\sqrt{\lambda_{1}}$ & $\sqrt{\lambda_{2}}$ & $\sqrt{\lambda_{3}}$ & $\sqrt{\lambda_{4}}$ \\
\hline 10 & 3.2999 & 5.8227 & 8.0566 & 9.9254 \\
20 & 3.3432 & 6.1715 & 8.9764 & 11.5700 \\
30 & 3.3517 & 6.2493 & 9.2172 & 12.0747 \\
40 & 3.3547 & 6.2777 & 9.3097 & 12.2799 \\
50 & 3.3561 & 6.2911 & 9.3540 & 12.3811 \\
\hline
\end{tabular}

Table 22: Response of frequency parameter with length-to-height ratio for CS case

\begin{tabular}{ccccc}
\hline$L / h$ & $\sqrt{\lambda_{1}}$ & $\sqrt{\lambda_{2}}$ & $\sqrt{\lambda_{3}}$ & $\sqrt{\lambda_{4}}$ \\
\hline 10 & 3.8908 & 6.4640 & 8.6451 & 10.4625 \\
20 & 3.9601 & 6.8823 & 9.6726 & 12.2367 \\
30 & 3.9738 & 6.9768 & 9.9456 & 12.7893 \\
40 & 3.9787 & 7.0114 & 10.0509 & 13.0153 \\
50 & 3.9809 & 7.0278 & 10.1016 & 13.1269 \\
\hline
\end{tabular}

Table 23: Response of frequency parameter with length-to-height ratio for $\mathrm{CC}$ case

\begin{tabular}{ccccc}
\hline$L / h$ & $\sqrt{\lambda_{1}}$ & $\sqrt{\lambda_{2}}$ & $\sqrt{\lambda_{3}}$ & $\sqrt{\lambda_{4}}$ \\
\hline 10 & 4.6747 & 7.1643 & 9.2702 & 11.0230 \\
20 & 4.7695 & 7.6618 & 10.4134 & 12.9352 \\
30 & 4.7883 & 7.7750 & 10.7211 & 13.5385 \\
40 & 4.7949 & 7.8166 & 10.8402 & 13.7864 \\
50 & 4.7980 & 7.8362 & 10.8976 & 13.9092 \\
\hline
\end{tabular}

Table 24: Response of frequency parameter with length-to-height ratio for CF case

\begin{tabular}{ccccc}
\hline$L / h$ & $\sqrt{\lambda_{1}}$ & $\sqrt{\lambda_{2}}$ & $\sqrt{\lambda_{3}}$ & $\sqrt{\lambda_{4}}$ \\
\hline 10 & 2.5825 & 4.6094 & 7.1657 & 9.2693 \\
20 & 2.5724 & 4.6967 & 7.6417 & 10.4000 \\
30 & 2.5705 & 4.7133 & 7.7491 & 10.7018 \\
40 & 2.5699 & 4.7191 & 7.7886 & 10.8183 \\
50 & 2.5696 & 4.7218 & 7.8071 & 10.8745 \\
\hline
\end{tabular}

\section{Mode Shapes}

Proper understanding of mode-shapes is an essential aspect of vibrational characteristics which helps in designing various engineering systems like nanoelectromechanical system. In this regards, mode-shapes are plotted in Figures 6-17 for different modes of SS, CS, CC, and CF. Mode-shapes are demonstrated by taking, $L=10, \mu=1$, $K_{w}=500$ and grid points $N=100$. These graphs are
Table 25: Response of frequency parameter with Winkler modulus parameter for SS case

\begin{tabular}{ccccc}
\hline$K_{w}$ & $\sqrt{\lambda_{1}}$ & $\sqrt{\lambda_{2}}$ & $\sqrt{\lambda_{3}}$ & $\sqrt{\lambda_{4}}$ \\
\hline 0 & 3.1187 & 6.1390 & 8.9659 & 11.5651 \\
50 & 3.3432 & 6.1715 & 8.9764 & 11.5700 \\
100 & 3.5299 & 6.2035 & 8.9869 & 11.5749 \\
200 & 3.8332 & 6.2661 & 9.0077 & 11.5847 \\
500 & 4.4661 & 6.4433 & 9.0693 & 11.6138 \\
1000 & 5.1457 & 6.7097 & 9.1693 & 11.6619 \\
\hline
\end{tabular}

Table 26: Response of frequency parameter with Winkler modulus parameter for CS case

\begin{tabular}{ccccc}
\hline$K_{w}$ & $\sqrt{\lambda_{1}}$ & $\sqrt{\lambda_{2}}$ & $\sqrt{\lambda_{3}}$ & $\sqrt{\lambda_{4}}$ \\
\hline 0 & 3.8320 & 6.8590 & 9.6642 & 12.2326 \\
50 & 3.9601 & 6.8823 & 9.6726 & 12.2367 \\
100 & 4.0769 & 6.9055 & 9.6809 & 12.2409 \\
200 & 4.2843 & 6.9511 & 9.6976 & 12.2491 \\
500 & 4.7727 & 7.0827 & 9.7471 & 12.2738 \\
1000 & 5.3547 & 7.2871 & 9.8279 & 12.3146 \\
\hline
\end{tabular}

Table 27: Response of frequency parameter with Winkler modulus parameter for CC case

\begin{tabular}{ccccc}
\hline$K_{W}$ & $\sqrt{\lambda_{1}}$ & $\sqrt{\lambda_{2}}$ & $\sqrt{\lambda_{3}}$ & $\sqrt{\lambda_{4}}$ \\
\hline 0 & 4.6981 & 7.6449 & 10.4067 & 12.9317 \\
50 & 4.7695 & 7.6618 & 10.4134 & 12.9352 \\
100 & 4.8379 & 7.6786 & 10.4201 & 12.9387 \\
200 & 4.9666 & 7.7119 & 10.4335 & 12.9457 \\
500 & 5.3023 & 7.8092 & 10.4733 & 12.9667 \\
1000 & 5.7507 & 7.9637 & 10.5387 & 13.0013 \\
\hline
\end{tabular}

Table 28: Response of frequency parameter with Winkler modulus parameter for CF case

\begin{tabular}{ccccc}
\hline$K_{w}$ & $\sqrt{\lambda_{1}}$ & $\sqrt{\lambda_{2}}$ & $\sqrt{\lambda_{3}}$ & $\sqrt{\lambda_{4}}$ \\
\hline 0 & 1.9098 & 4.6213 & 7.6246 & 10.3932 \\
50 & 2.5724 & 4.6967 & 7.6417 & 10.4000 \\
100 & 2.9356 & 4.7686 & 7.6587 & 10.4068 \\
200 & 3.4102 & 4.9034 & 7.6924 & 10.4203 \\
500 & 4.2233 & 5.2523 & 7.7909 & 10.4604 \\
1000 & 4.9960 & 5.7139 & 7.9473 & 10.5263 \\
\hline
\end{tabular}

plotted by varying non-uniform parameter $\eta$ as $-2,0$ and 2. From these figures, we may note that the amplitudes of displacements are affected significantly by varying nonuniform parameters. 
(a) SS nanobeam

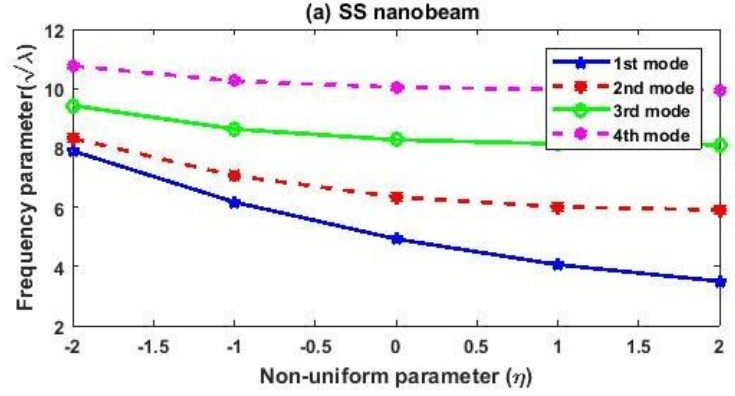

(c) CC nanobeam

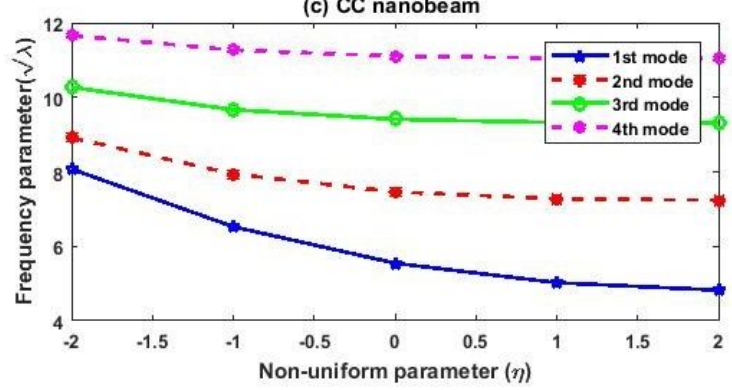

Figure 3: Frequency parameters Vs. non-uniform parameters

(a) SS nanobeam

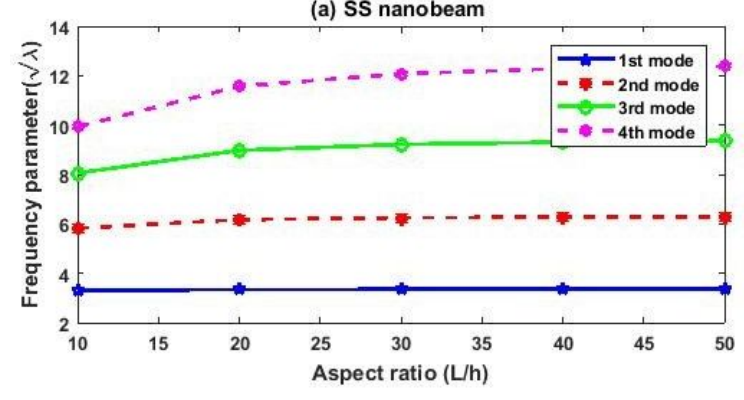

(c) CC nanobeam

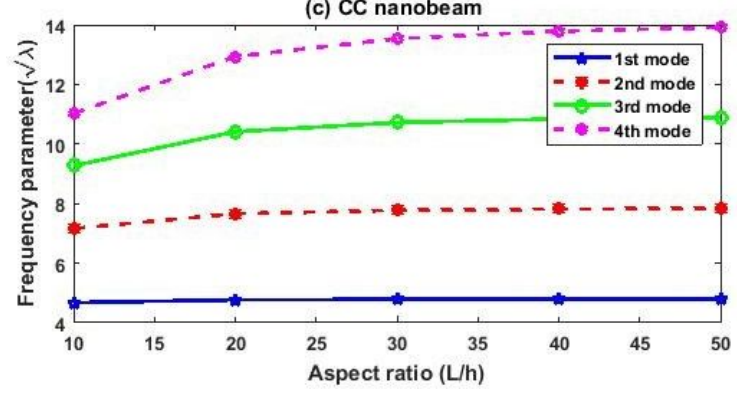

Figure 4: Frequency parameters Vs. Aspect ratio (b) CS nanobeam

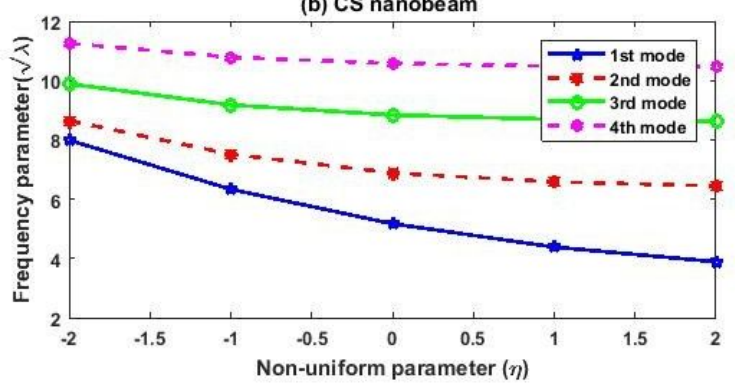

(d) CF nanobeam

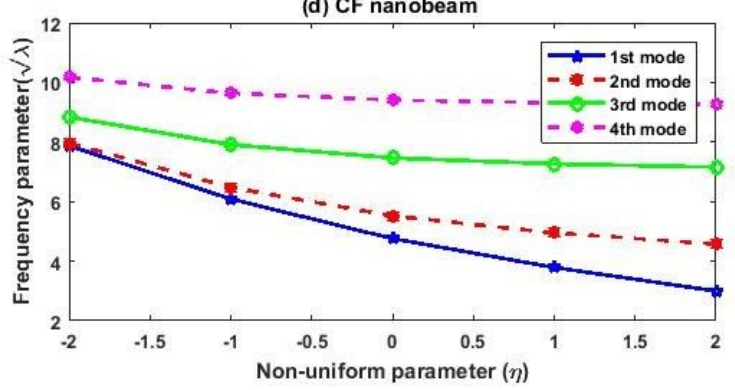


(a) SS nanobeam

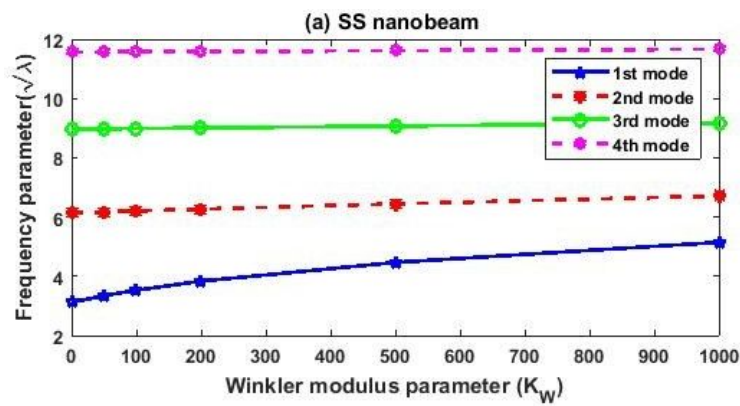

(c) CC nanobeam

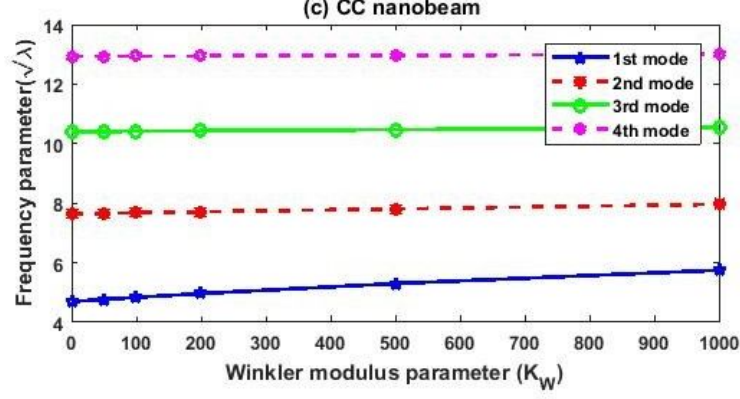

Figure 5: Frequency parameters Vs. Winkler modulus parameters

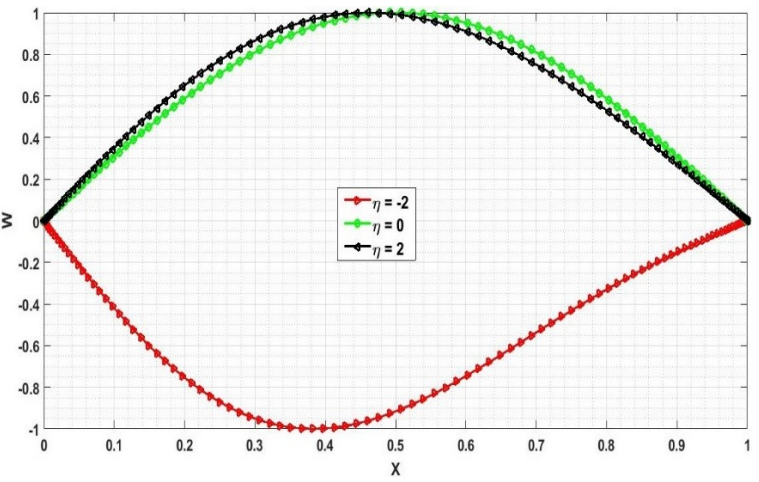

Figure 6: SS-1 ${ }^{\text {st }}$ Mode

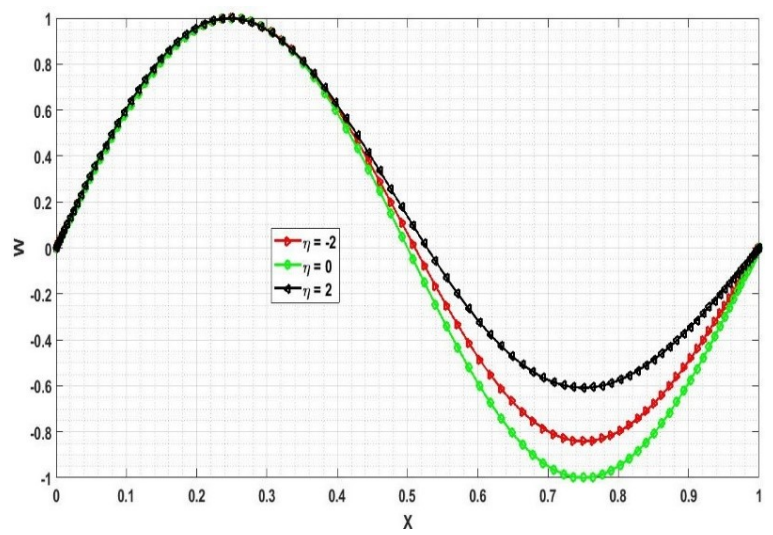

Figure 7: SS-2 ${ }^{\text {nd }}$ Mode

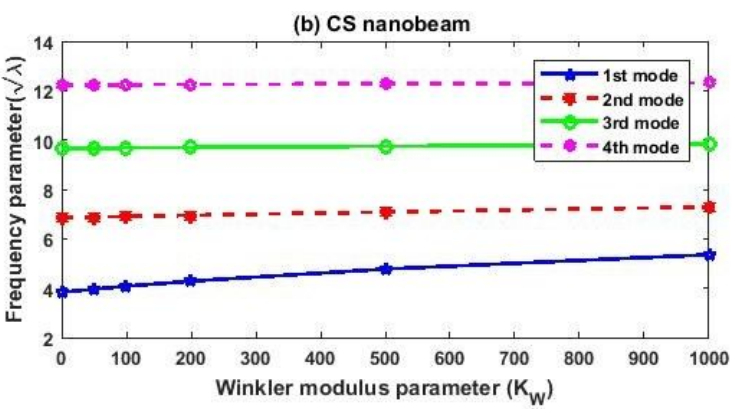

(d) CF nanobeam
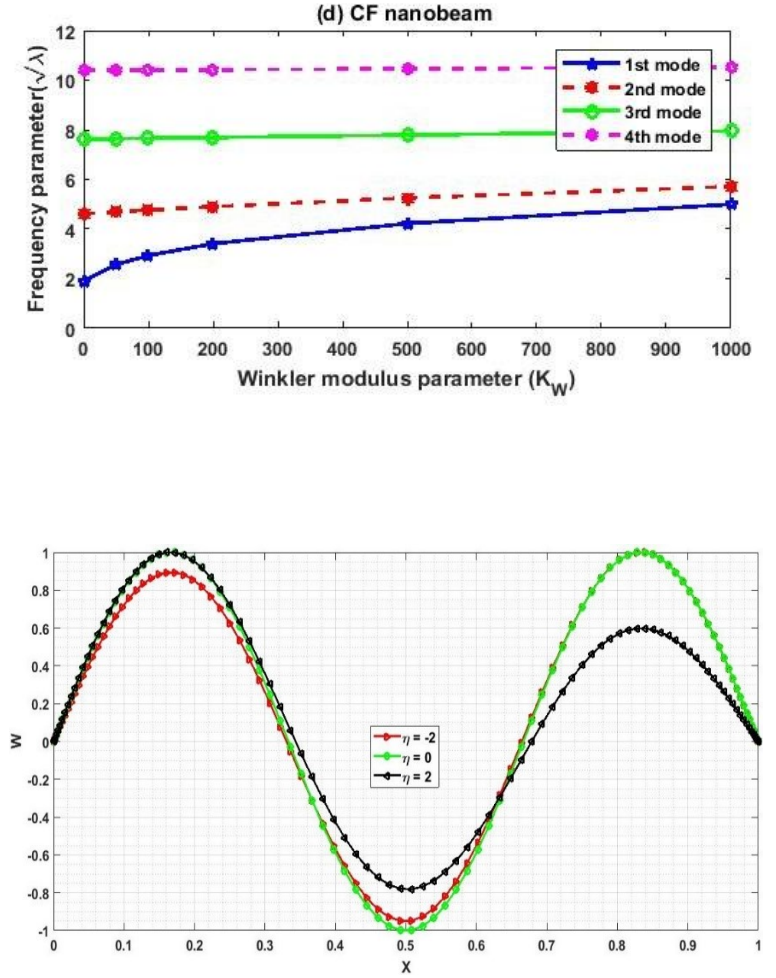

Figure 8: SS-3 $3^{\text {rd }}$ Mode

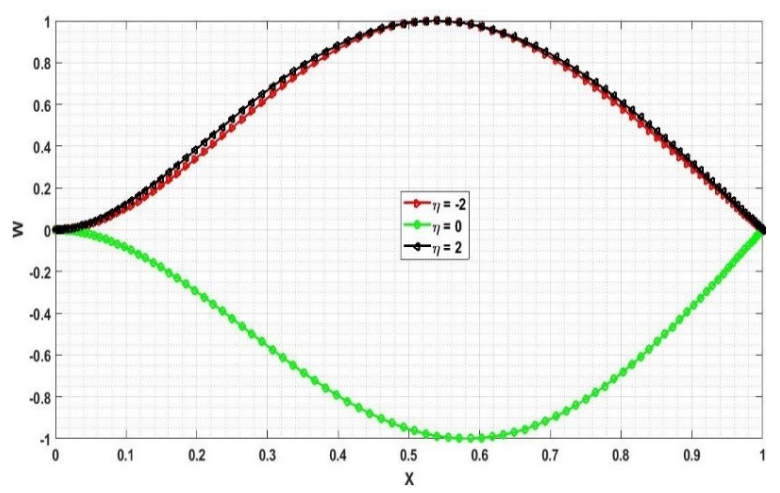

Figure 9: $\mathrm{CS}-1^{\text {st }}$ Mode

\section{Conclusions}

Present investigation deals with the study of free vibration of variable cross-section single-layered graphene nano- 


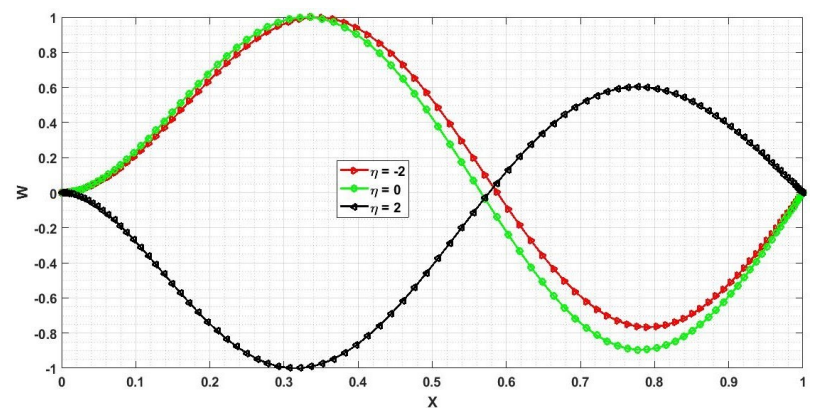

Figure 10: $C S-2^{\text {nd }}$ Mode

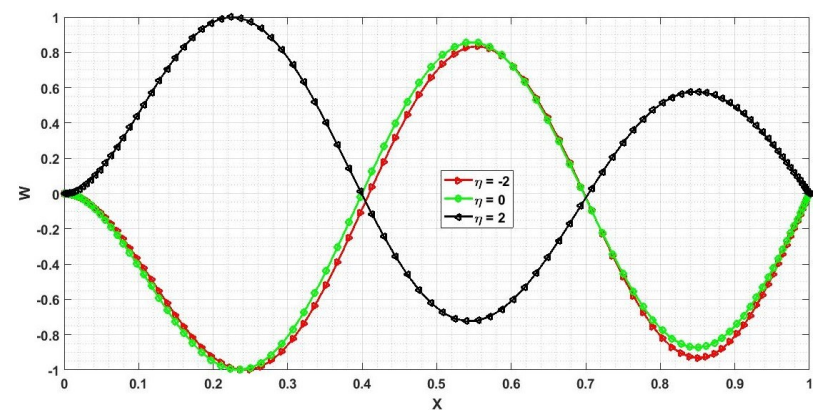

Figure 11: $\mathrm{CS}-3^{\text {rd }}$ Mode

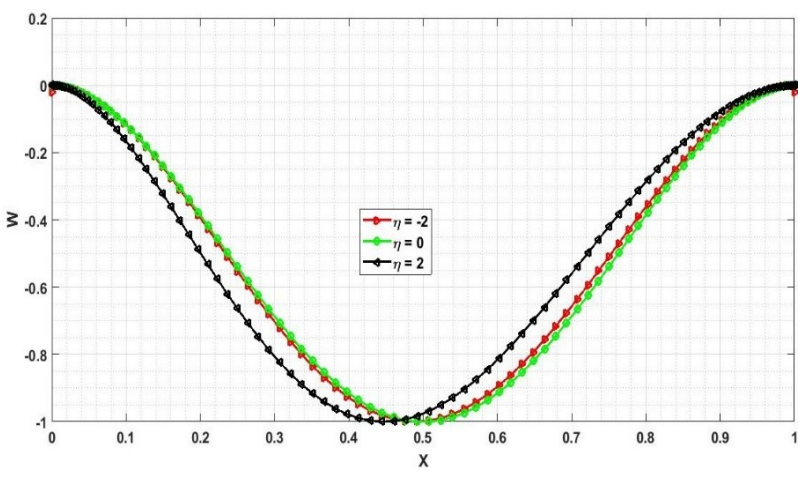

Figure 12: $\mathrm{CC}-1^{\text {st }}$ Mode

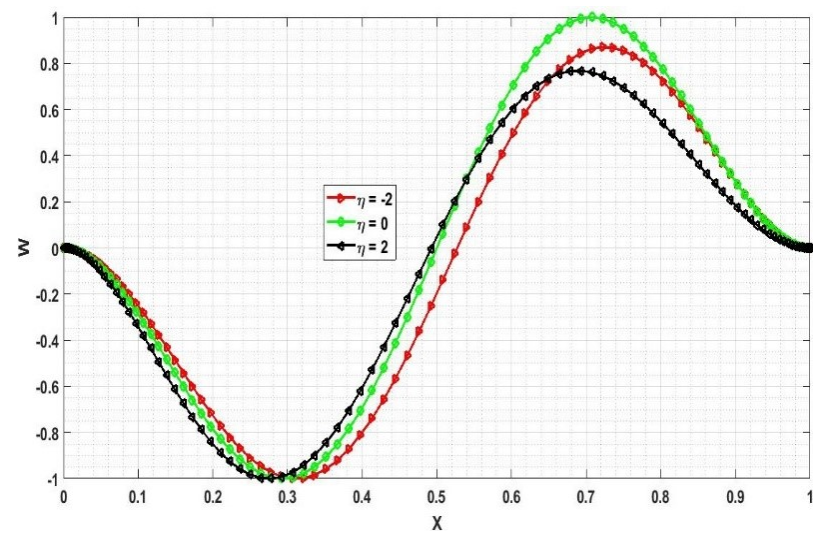

Figure 13: $C C-2^{\text {nd }}$ Mode

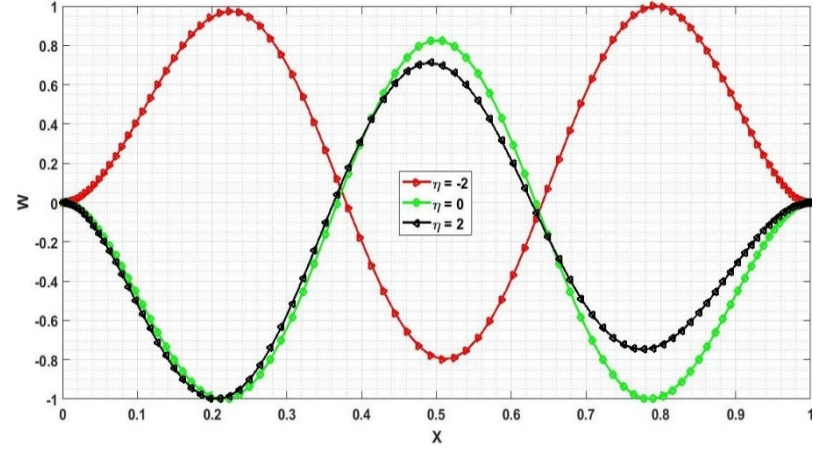

Figure 14: $C C-3^{\text {rd }}$ Mode

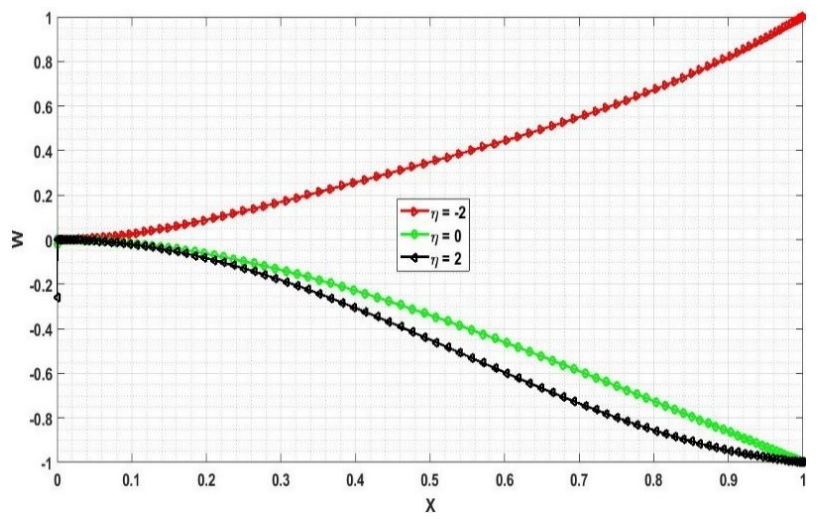

Figure 15: CF-1 ${ }^{\text {st }}$ Mode

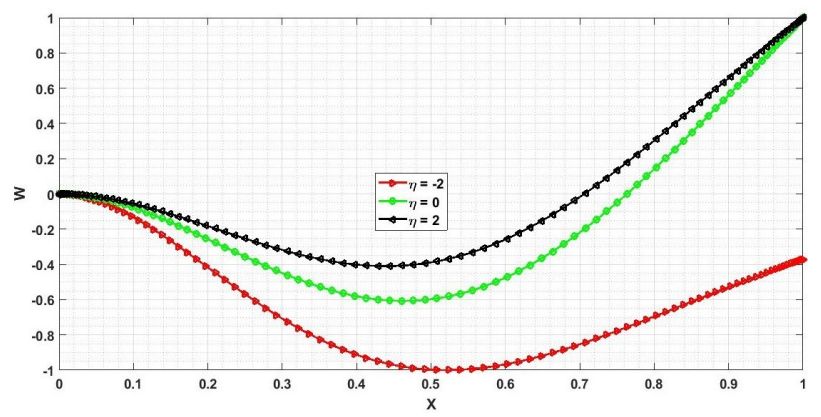

Figure 16: $C F-2^{\text {nd }}$ Mode

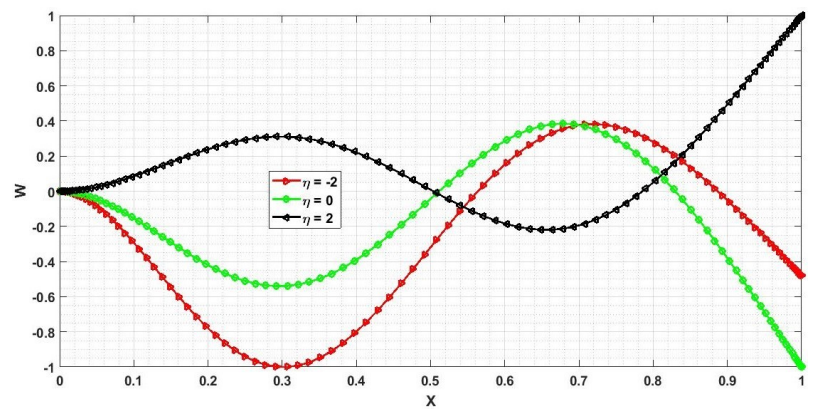

Figure 17: CF- $3^{\text {rd }}$ Mode 
ribbons (SLGNRs) which is placed on Winkler elastic foundation using the Differential Quadrature Method (DQM). Eringen's nonlocal theory is employed along with EBT for this study. The tabular and graphical results are obtained by solving the generalized Eigen value problem using MATLAB codes. The impacts of several scaling parameters such as the Winkler modulus parameter, non-uniform parameter, nonlocal parameter, and aspect ratio are studied. Following are the main observations.

- The frequency parameter decreases with increase in the non-uniform parameter and this decrease is more significant in the case of higher modes.

- The Clamped-Clamped case possesses the highest frequency parameters whereas the Clamped-Free case possesses the lowest among all other types of boundary conditions.

- The effect of the nonlocal parameter is more significant in higher modes. Frequency parameters decrease with increase in nonlocal parameter except fundamental frequency parameter of CF case.

- Frequency parameters with increase in aspect ratio for all the mode of all the standard boundary conditions except 1st mode of C-F case, where it is just reverse.

- The frequency parameter increases with increase in the Winkler modulus parameter and this increase is more significant in the case of lower modes.

- The amplitudes of displacements are affected significantly by varying non-uniform parameters.

Acknowledgement: The authors would like to thank Defence Research \& Development Organization(DRDO), Ministry of Defence, New Delhi, India (Sanction Code: DG/TM/ERIPR/GIA/17-18/0129/020) for the funding to carry out the present research work.

\section{References}

[1] A. K. Geim, K. S. Novoselov (2007) The rise of graphene, Nature Materials, Vol-6, p. 183-191.

[2] A. K. Geim (2009) Graphene: status and prospects, Science, Vol324, p. 1530-1534.

[3] K. S. Novoselov, V. I. Fal'ko, L. Colombo, P. R. Gellert, M. G. Schwab, K. A. Kim (2012) Roadmap for graphene, Nature, Vol490, p. 192-200.

[4] M. Fujita, K. Wakabayashi, K. Nakada, K. Kusakabe (1996) Peculiar Localized State at Zigzag Graphite Edge, Journal of the Physics Society Japan, Vol-65, p. 1920.

[5] K. Nakada, M. Fujita, G. Dresselhaus, M.S. Dresselhaus (1996) Edge state in graphene ribbons: Nanometer size effect and edge shape dependence, Physical Review B, Vol- 54, p. 17954.
[6] K. Wakabayashi, M. Fujita, H. Ajiki, M. Sigrist (1999) Electronic and magnetic properties of nanographite ribbons, Physical Review B, Vol-59, p. 8271.

[7] A.C. Eringen (1972) Nonlocal polar elastic continua, Internat. J. Engrg. Sci., Vol-10, Issue-1, p.1-16.

[8] F. Tornabene, N. Fantuzzi, M. Bacciocchi, E. Viola (2016) Effect of agglomeration on the natural frequencies of functionally graded carbon nanotube-reinforced laminated composite doubly-curved shells, Composites Part B, Volume 89, p.187-218.

[9] N. Fantuzzi, F. Tornabene, M. Bacciocchi, R. Dimitri (2017) Free vibration analysis of arbitrarily shaped Functionally Graded Carbon Nanotube-reinforced plates, Composites Part B, Vol-115, p. 384-408.

[10] F. Tornabene, N. Fantuzzi, M. Bacciocchi (2017) Linear static response of nanocomposite plates and shells reinforced by agglomerated carbon nanotubes, Composites Part B Vol-115, p. 449-476.

[11] D. Bani'c, M. Bacciocchi, F. Tornabene, A. J. M. Ferreira (2017) Influence of Winkler-Pasternak Foundation on the Vibrational Behavior of Plates and Shells Reinforced by Agglomerated Carbon Nanotubes, Applied Sciences, Vol-7, p. 1-55.

[12] S.K. Jena, S. Chakraverty (2018) Free Vibration Analysis of Single Walled Carbon Nanotube with Exponentially Varying Stiffness, Curved and Layered Structures, Vol-5(1), p.201-212.

[13] S.K. Jena, S. Chakraverty (2018) Free Vibration Analysis of Variable Cross-Section Single Layered Graphene Nano-Ribbons (SLGNRs) Using Differential Quadrature Method, Frontiers in Built Environment, Vol-4, p.63.

[14] S.K. Jena, S. Chakraverty (2018) Free vibration analysis of EulerBernoulli nanobeam using differential transform method, International Journal of Computational Materials Science and Engineering, Vol-7(3), p.1850020.

[15] S.K. Jena, S. Chakraverty (2018) Free Vibration of Single Walled Carbon Nanotube Resting on Exponentially Varying Elastic Foundation, Curved and Layered Structures, Vol-5(1), p.260-272.

[16] S.K. Jena, S. Chakraverty, R.M. Jena, F. Tornabene (2019)A novel fractional nonlocal model and its application in buckling analysis of Euler-Bernoulli nanobeam, Materials Research Express, Vol6(5), p.055016

[17] A. Mirzabeigy (2014) Semi-analytical approach for free vibration analysis of variable cross-section beams resting on elastic foundation and under axial force, International Journal of Engineering, Transactions C: Aspects, Vol- 27, p. 455-463.

[18] T. Chaudhari, S. Maiti (1999) Modelling of transverse vibration of beam of linearly variable depth with edge crack, Engineering Fracture Mechanics, Vol- 63, p. 425-445.

[19] M.C. Ece, M. Aydogdu, V. Taskin (2007) Vibration of a variable cross-section beam, Mechanics Research Communications, Vol34, p. 78-84.

[20] R. Attarnejad, A. Shahba (2011) Dynamic basic displacement functions in free vibration analysis of centrifugally stiffened tapered beams; a mechanical solution, Meccanica, Vol- 46, p. 1267-1281.

[21] J. Peddieson, G.R. Buchanan, R.P. McNitt (2003) Application of nonlocal continuum models to nanotechnology, International Journal of Engineering Science, Vol-41, p. 305-312.

[22] C. Wang, Y. Zhang, X. He (2007) Vibration of nonlocal timoshenko beams, Nanotechnology, Vol-18, p. 105-113.

[23] J.N. Reddy (2007) Nonlocal theories for bending, buckling and vibration of beams, Internat. J. Engrg. Sci., Vol-45, Issue- 2, p. 288-307. 
[24] M. Aydogdu (2009) A general nonlocal beam theory: its application to nanobeam bending, buckling and vibration, Physica E, Vol- 41, Issue -9, p. 1651-1655.

[25] M.A. Eltaher, Amal E. Alshorbagy, F.F. Mahmoud (2013) Vibration analysis of Euler-Bernoulli nanobeams by using finite element method, Applied Mathematical Modelling, Vol-37, Issue-7, p.4787-4797.

[26] S. Hosseini Hashemi, H. Bakhshi Khaniki (2016) Analytical solution for free vibration of a variable cross-section nonlocal nanobeam, International Journal of Engineering, Vol-29, p. 688 696.

[27] M.I. Gorbunov-Posadov, T.A. Malikova (1973) The design of structures on an elastic foundation, Stroiizdat, Moscow.

[28] K. Al-Hosani, S. Fadhil, A. El-Zafrany (1999) Fundamental solution and boundary element analysis of thick plates on Winkler foundation, Computers and Structures, Vol-70, p.325-336.

[29] M. HetVenyi (1961) Beams on elastic foundations. University of Michigan Press, Ann Arbor.

[30] G. Gazetas, G. Mylonakis (1998) Seismic soil-structure interaction: new evidence and emerging Issues, Geotechnical Special Publication, Vol-75, p.1119-1174.

[31] K.P. Soldatos, A. P. S. Selvadurai (1985) Flexure of beams resting on hyperbolic elastic foundations, International Journal of Solids Structures, Vol- 21, p. 373-388.

[32] J.S. Smail (1991) Large deflection response of annular plates on Pasternak foundations. International Journal of Solids Structures, Vol-27, p.1073-1084.

[33] K. K. Raju, G. V. Rao (1993) Effect of a non-linear elastic foundation on the mode shapes in stability and vibration problems of uniform columns/beams, Journal of Sound and Vibration, Vol160, p.369-371.

[34] Y. S. Shih, B. T. Blotter (1993) Non-linear vibration analysis of arbitrarily laminated thin rectangular plates on elastic foundations, Journal of Sound and Vibration, Vol-167, p.433-459.

[35] D. Zhou (1993) A General solution to vibrations of beams on variable Winkler elastic foundation, Computers \& Structures, Vol-47, p.83-90.

[36] M. Eisenberger (1994) Vibration frequencies for beams on variable one- and two-paramter elastic foundations, Journal of Sound and Vibrations, Vol-176, p.577-584.

[37] L. Auersch (2008) Dynamic interaction of various beams with the underlying soil -finite and infinite, half-space and Winkler models, European Journal of Mechanics A/Solids, p.27933-958.
[38] M. Eisenberger, J. Clastornik (1987) Vibrations and buckling of a beam on a variable Winkler elastic foundation, Journal of Sound and Vibration, Vol-115, p.233-241.

[39] P. Ruge, C. Birk (2007) A comparison of infinite Timoshenko and Euler-Bernoulli beam models on Winkler foundation in the frequency- and time-domain, Journal of Sound and Vibration, Vol-304, p.932-947.

[40] H.P. Lee (1998) Dynamic response of a Timoshenko beam on a Winkler foundation subjected to a moving mass, Applied Acous tics, Vol- 55, p.203-215.

[41] M. H. Huang, D.P. Thambiratnam (2001) Deflection response of plate on Winkler foundation to moving accelerated loads, Engineering Structures, Vol-23, p.1134-1141.

[42] H. R. Öz, M. Pakdemirli (2006) Two-to-one internal resonances in a shallow curved beam resting on an elastic foundation, Acta Mechanica, Vol-185, p. 245-260.

[43] X. Ma, J.W. Butterworth, G.C. Clifton (2009) Static analysis of an infinite beam resting on a tensionless Pasternak foundation, European Journal of Mechanics A/Solids, Vol-28, p.697-703.

[44] O. Civalek (2007) Nonlinear analysis of thin rectangular plates on Winkler-Pasternak elastic foundations by DSC-HDQ methods, Applied Mathematical Modeling, Vol-31, p. 606-624.

[45] A Kacar, HT Tan, MO Kaya (2011) Free vibration analysis of beams on variable winkler elastic foundation by using the differential transform method, Mathematical computational applications, Vol. 16, p. 773-783.

[46] S Chakraverty and Laxmi Behera (2015) Vibration and buckling analyses of nanobeams embedded in an elastic medium, Chinese Physics B, Volume 24, p. 097305(1-8).

[47] Ö. Civalek (2004) Application of differential quadrature (DQ) and harmonic differential quadrature (HDQ) for buckling analysis of thin isotropic plates and elastic columns, Engineering Structures, Vol-26, p. 171-186.

[48] Ö. Civalek (2005) Geometrically nonlinear dynamic analysis of doubly curved isotropic shells resting on elastic foundation by a combination of harmonic differential quadrature-finite difference methods, International Journal of Pressure Vessels and Piping, Vol-82, p. 470-479.

[49] R. Bellman, J. Casti, Differential quadrature and long-term integration, J. Math. Anal. Appl., Vol- 34, Issue-2, 1971, p. 235-238.

[50] J. Quan, C. Chang (1989) New insights in solving distributed system equations by the quadrature method-I. Analysis, Comput. Chem. Eng., Vol-13, Issue- 7, p. 779-788.

[51] C. Shu, Differential Quadrature and its Application in Engineering, Springer, 2000. 